\title{
Empirically derived climate predictability over the extratropical northern hemisphere
}

\author{
J.B. Elsner' and ALA. Tsonis' \\ ${ }^{1}$ Dept. of Meteorology, Florida State University, Tallahassee, FL 32306, USA \\ ${ }^{2}$ Dept. of Geosciences, University of Wisconsin-Milwaukee, Milwaukee, WI 53201, USA \\ Received 1 December 1993 - Accepted 16 February 1994 - Communicated by A.D. Kirwan
}

\begin{abstract}
A novel application of a technique developed from chaos theory is used in describing seasonal to interannual climate predictability over the Northern Hemisphere (NH). The technique is based on an empirical forecast scheme-local approximation in a reconstructed phase space-for time-series data. Data are monthly $500 \mathrm{hPa}$ heights on a latitude-longitude grid covering the $\mathrm{NH}$ from $20^{\circ} \mathrm{N}$ to the equator. Predictability is estimated based on the linear correlation between actual and predicted heights averaged over a forecast range of one- to twelve-month lead. The method is capable of extracting the major climate signals on this time scale including ENSO and the North Atlantic Oscillation.
\end{abstract}

\section{Introduction}

The Earth's global atmosphere displays a tremendous amount of variability on many space and time scales. As a result of sensitive dependence on initial conditions, all forecasts of this atmospheric variability are accompanied by error growth which leads to limits on accurate predictions. The question of just how predictable the weather and climate are can be addressed using observations or numerical model simulations. The present study examines the problem of seasonal to interannual climate predictability using a nonlinear time-series model on observed data. The approach centers on building a model from earlier data and comparing model predictions with data later in the record. Predictability is assessed by deterioration of forecast skill as predictions are made further into the known future. The appealing aspect of this approach is that it is independent of forecasting method, at least in theory.

\section{Data and Methodology}

Consider 522 consecutive maps of monthly-averaged 500 $\mathrm{hPa}$ geopotential heights over the extratropical Northern Hemisphere, where each map consists of a latitudelongitude grid (18 latitude points between $20^{\circ} \mathrm{N}$ and the pole by 60 longitude points around each latitude circle). Geopotential heights at a given pressure level indicate the density in the underlying atmosphere and horizontal gradients of the height field approximate the horizontal momentum field. Data are from the US National Meteorological Center's (NMC's) initial analyses for the period January 1946 through June 1989. Thus, at each latitude-longitude grid point there exists a univariate time series of geopotential heights for 522 consecutive months. A nonlinear forecast model is trained on the first 480 months ( 40 years; the training set) of each time series separately and a relative measure of predictability is assessed by examining linear correlations between actual and predicted values over the remaining 42 months (3.5 years; the target set).

The univariate nonlinear prediction model is a version of the Farmer and Sidorowich (1987) and Sugihara and May (1990) interpolative algorithm used in Wales (1991) and is based on the simplex method in phase space. An embedding dimension (E) is chosen and the phase space constructed using the method of time delays. Based on the decay of the autocorrelation function a time delay of two months is used, however, results are not sensitive to the exact choice of a time delay in the range of one 


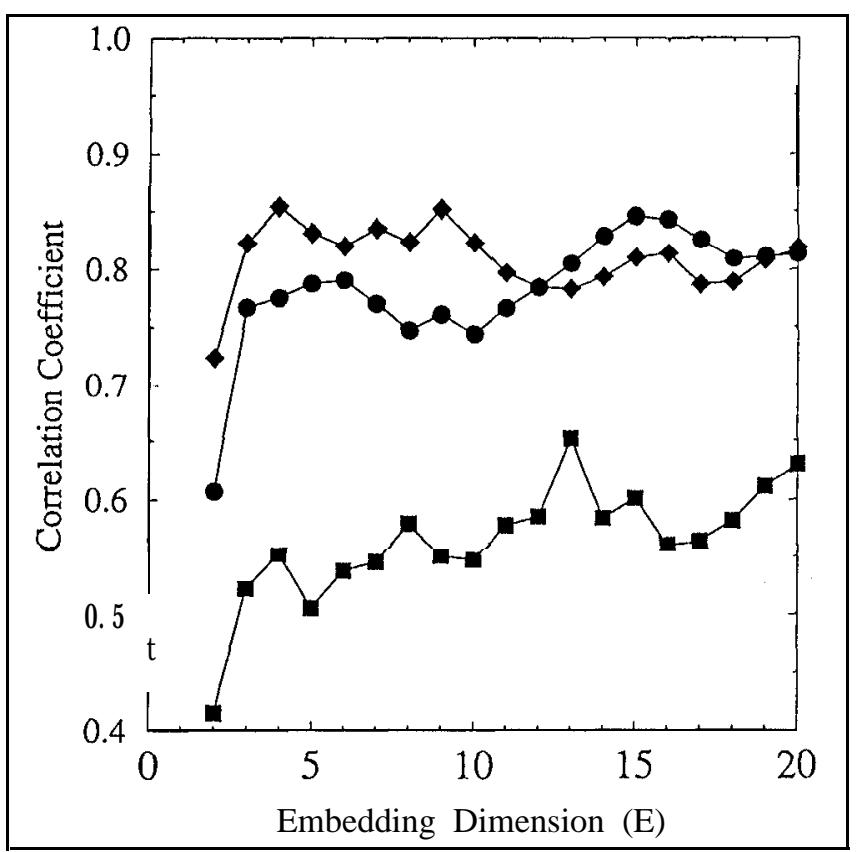

Figure 1. Prediction error versus embedding dimension. The prediction model is a local-linear approximation method in phase space. Prediction error is estimated by the linear correlation between actual and predicted values for a one-month forecast of 500 $\mathrm{hPa}$ geopotential heights. Shown are prediction errors for three representive grid points over the Northern Hemisphere (grid point $(1,18)$ with circles, grid point $(45,1)$ with squares, and grid point $(31,12)$ with diamonds).

to twelve months. For each point in phase space, nearby points are located and a minimal neighborhood is defined such that the sequence containing the target point is located within the simplex with a minimum diameter formed from the $\mathbf{E}+1$ closest neighbors. A one-month prediction is made by keeping track of where the points in the simplex end up after one month into the known future. The Pearson's product-moment linear correlation coefficient is computed over all target points to quantify predictability. The predictability field is then contoured on a map of the extratropical Northern Hemisphere.

The nonlinear prediction model requires the specification of an optimum embedding dimension. To do this Sugihara and May (1990) suggest plotting the one-step (one month) prediction error as a function of embedding dimension. The results of such a procedure for three representative grid points over the $\mathrm{NH}$ (the 1,1 grid point is located at $20^{\circ} \mathrm{N}$ and $0^{\circ} \mathrm{E}$ with indices increasing to the north and east) is shown in Figure 1. For all grid points linear correlation between actual and predicted values increase from low embeddings and saturate above embedding dimension four. Therefore, an embedding dimension of five is used for all grid points.

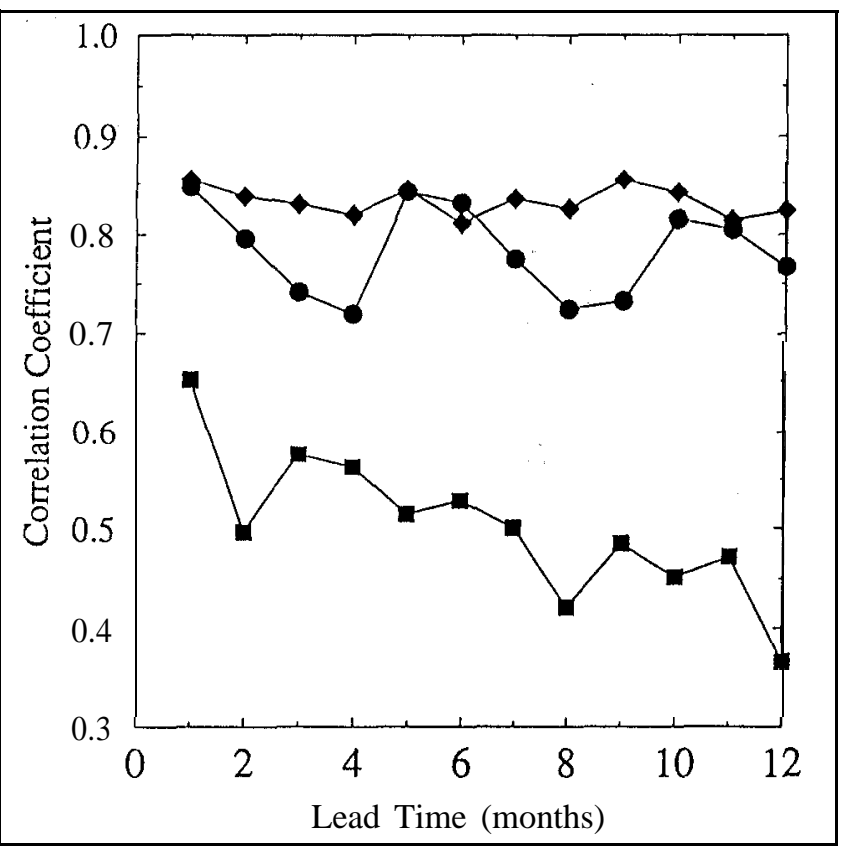

Figure 2. Prediction error versus lead time for the same three grid points used in Figure 1. The prediction model is a local-linear approximation method in phase space with an embedding dimension of five months. Prediction error is estimated by the linear correlation between actual and predicted values for predictions made from one- to twelve-month lead.

Results are not sensitive to embedding dimensions in the range from two to eight.

Predictions over the target set are made at each grid point for lead times from one to twelve months. Examples of forecast skill over this period are shown in Figure 2 for the same points used in Figure 1. Note that a twomonth forecast is made by considering the one-month prediction value rather than the known value for that month; and the same procedure is followed for all forecasts out to twelve months. These twelve forecast errors (one error for each month) are averaged to obtain a single prediction error representing how well the time series at each point can be predicted on average over an entire year.

\section{Results and Discussion}

Linear correlations (X 100) between actual and predicted $500 \mathrm{hPa}$ geopotential heights averaged over the 42-month (January 1986 - June 1989) target set and averaged over lead times of one through twelve months for each grid point are color contoured in Figure 3. Correlations range from 0.5 (violet) to 0.9 (red). Results have been smoothed using a nine-point uniform filter. 


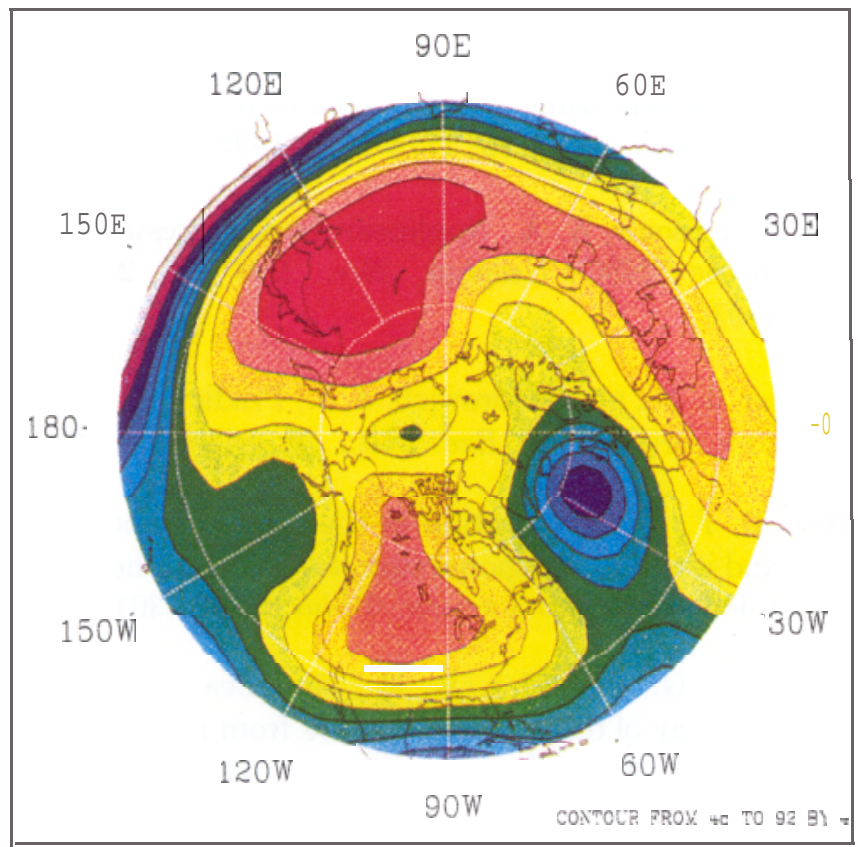

Figure 3. Linear correlations (X 100) between actual and predicted $500 \mathrm{hPa}$ geopotential heights averaged over the 42 month (January 1986 - June 1989) target set and averaged over oneto twelve-month forecasts for each latitude-longitude grid point. Correlations range from 0.5 (violet) to 0.9 (red). Results have been smoothed using a nine-point uniform filter. Spatial heteorogeneity of seasonal to interannual predictability over the extratropical Northern Hemisphere is clearly evident.

Spatial heteorogeneity of seasonal to interannual predictability over the extratropical $\mathrm{NH}$ is clearly evident. In general best predictions are found over midlatitude continental regions with poorer predictions over oceanic regions and at lower latitudes.

The explanation for the spatial variability of predictability can be related, at least in part, to the strength of the seasonal cycle. The seasonal cycle, which is predictable, is most pronounced over midlatitude continental regions and less so over the oceans and at lower latitudes. Predictability of the seasonal cycle over continental regions can be explained by the strong dependence of lower tropospheric temperatures (and thus density) to changes in solar insolation. This dependence is much weaker over large ocean regions where sensible heat from the waters can significantly modulate temperatures in the lower troposphere. Of special interest is the very poor predictive skill over portions of the North Atlantic Ocean and over the subtropical western Pacific Ocean.

The lack of prediction skill over the North Atlantic Ocean may be related to blocking episodes commonly found in this region (Rex, 1950; Blackmon, 1976; Dole,
1986; Barnston and Livezey, 1987; Elsner, 1992). Blocking can last for several weeks and interrupt the normal west to east flow of weather systems thus producing large-scale temperature anomalies. Explanation for initiation and maintenance of blocking episodes is still largely speculative but may center on the slowly varying atmospheric boundary conditions of the underlying oceans. Strong aperiodically occurring blocking episodes can significantly alter the normal progression of seasons and thus effect the ability to forecast seasonal to interannual climate changes.

Over the western Pacific Ocean in the subtropics, poor forecast skill is attributable to the seasonal to interannual "footprint" of the chaotic El-Niñol - Southern Oscillation (ENSO) signal (Vallis, 1986; Tsonis and Elsner, 1992; Elsner and Tsonis, 1993). ENSO represents a coupling between the tropical atmosphere and ocean over the Pacific Basin. Extended periods of anomalously warm sea-surface temperatures occurring off the coast of South America (El-Nino) are linked to the zonal circulation of atmospheric mass (Southern Oscillation). The variability of this coupling between sea surface temperatures and lower tropospheric pressures in the tropical Pacific makes accurate prediction on the seasonal time scale difficult. This region of low predictability has spatial coherence extending into the extratropical Northern Hemisphere.

\section{Conclusions}

This short report describes a novel application of a technique from nonlinear dynamical systems which is used in describing short-term climate predictability. The technique centers around the use of a nonlinear forecasting scheme (local approximation in phase space) for timeseries data. Data are taken from NMCs initial analyses. Predictability is measured as the linear correlation between actual and predicted $500 \mathrm{hPa}$ geopotential heights over a 42-month target set of the time record and over forecasts ranging from one to twelve-month lead. Model training is performed on an earlier 40-year sample. The predictability field is contoured on a map of the extratropical Northern Hemisphere.

From the results it is concluded that predictability on the seasonal to interannual time scale is closely tied to the seasonal cycle. Predictability is enhanced over continental regions of the middle latitudes where the seasonal cycle is most pronounced as a result of the dependence of lower tropospheric heating on the amount of solar insolation. Forecast skill is considerably less over the oceans and at lower latitudes where sensible heat in the 
former and less variation in insolation in the latter are important in modulating the progression of seasons. The well-documented blocking episodes over the North Atlantic Ocean and the ENSO over the Pacific are conspicuous in producing regions of poor short-term climate predictability over portions of the Northern Hemisphere. Reasons are attributed to the sensitive nature of the coupling between the underlying oceans and the atmosphere as well as to the modulation of the seasonal cycle. Based on the fact that the present results match very well with previous regional studies of climate predictability it is concluded that this type of empirical analysis can prove very useful for studying global weather and climate predictability.

A cknowledgements. Thanks are extended to Jason C. Hess for his help with the color graphics and manuscript preparation.

\section{References}

Barnston, A. G., and Livezey, R. E., Classification, seasonality, and persistence of low-frequency atmospheric circulation patterns, Mon. Wea. Rev., 115, 1083-1126, 1987.

Blackmon, M. L., A climatological spectral study of the $500 \mathrm{mb}$ geopotential height of the northern hemisphere, J. Atmos. Sci., 33, 1607-1623, 1976.

Dole, R. M., Persistent anomalies of the extratropical northern hemisphere wintertime circulation: Structure, Mon. Wea. Rev., 114, 178-207, 1986.
Elsner, J. B., A description of low-frequency atmospheric oscillations over the extratropical northern hemisphere using singular spectrum analysis, Geophys. Res. Lett., 19, 1775-1778, 1992.

-, and Tsonis, A. A., Nonlinear dynamics established in the ENSO, Geophys. Res. Lett., 20, 213-216, 1993.

Farmer, J. D., and Sidorowich, J. J., Predicting chaotic time series, Phys. Rev. Lett., 59, 845-848, 1987.

Rex, D. F., Blocking action in the middle troposphere and it effect on regional climate. Part II: The climatology of blocking action, Tellus, 2, 275-301, 1950.

Sugihara, G., and May, R. M., Nonlinear forecasting as a way of distinguishing chaos from measurement error in time series, Nature, 344, 734-741, 1990.

Tsonis, A. A., and Elsner, J. B., Nonlinear prediction as a way of distinguishing chaos from random fractal sequences, Nature, 358, 217-220, 1992.

Vallis, G. K., El-Niño: A chaotic dynamical system? Science, 232, 243-245, 1986.

Wales, D. J., Calculating the rate of loss of information from chaotic time series by forecasting, Nature, 350, 485-488, 1991. 